\title{
Internet of Things Based Emotional Sensor System to Predict the Patient's Information Using Bioscience Technology
}

\author{
S. Brindha \\ CSA Department, St.Peter's Institute of Higher Education and \\ Research, Avadi, Tamil Nadu 600054, India \\ Corresponding author email: brindhas.mca@spiher.ac.in
}

\begin{abstract}
Today we are living in the modern machine world, which leads gradual increase of diseases in the real human world. The number of person who works with computer or electronic devices are more in the recent decades. Due to the usage of electronic device, people are affected mentally rather than physical. Determine the emotion of the people while working with computers or any electronic devices termed as blue eye technology. This technology used to predict the emotion of the user such as joy, sad, happy or disgust. This paper mainly focused on remote monitoring system of the patient information using Blue Eye technology with IoT. Physicians can directly monitor the patient emotional information using Emotional Sensor system in terms of blue eye technology which act as an important fact of bio science. The Analog data retrieved form the emotional sensor are transmitted to cloud database in the digital form. These digital data will be predicted with K-NN algorithm to determine the patient's information. This paper concluded that an emotional sensor system used to predict the patient's information in the bioscience technology would be effective. This proposed work monitor both the emotional information and also physical information in terms of heart beat, pressure etc. This proposed work will provide an effective and efficient result in monitoring the patient information using bio science with Iot.
\end{abstract}

KEY WORDS: BLUE EYE TECHNOLOGY, IOT, EMOTIONAL SENSOR, BIO-SCIENCE, PATIENTS INFORMATION.

\section{INTRODUCTION}

Blue Eye Technology is the combination terms of Blue tooth + Eye technology. Blue tooth termed as wireless technology to transmit the analog information to digital information. Eye referred as seeking of information retrieved by the sensors. This Blue Eye Technology named as emotional sensor which used to determine the patient's information using Wireless technology. This technology will improve the performance of the patients. Various Methodology used in predict the patient's information through emotional sensors. Three types

Biosc Biotech Res Comm P-ISSN: 0974-6455 E-ISSN: 2321-4007

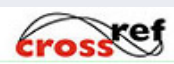

Identifiers and Pagination

Year: 2021 Vol: 14 No (6) Special Issue

Pages: 469-471

This is an open access article under Creative

DOI: $h t t p: / / d x$.doi.org/10.21786/bbrc/14.7.97
Commons License Attribn 4.0 Intl (CC-BY). of emotional sensors were used in blue eye technology such as a) Hand Emotional Sensor b) Eye Emotional Sensors and c) Voice Emotional Sensors.

Emotional Sensor further classified into two types of sensor such as emotion mouse sensor and Semitic mouse. Brain Computer Interface which act as intermediate between the patients details and transmit the information from analog to digital in the cloud environment through wireless devices. Semitic mouse further classified into three types based on the eyes such as a) expression glass b) magic pointing and c) eye tracking.

Literature Review: Blessed et al., (2018) attempts to deliver statistics approximately Blue eyes technological expertise in current engineering. It is based on tough facts, research and studies accrued from unique authors, books, journals, documents from internet web sites in desire that it's going to function a sufficient mastering aid for supposed readers. The paper noted Blue eyes technological Blessed et al., (2018) know-how to beneficial aid appreciation and
Article Information

Received: $19^{\text {th }}$ Aug 2021

ccepted after revision: $17^{\text {th }}$ Oct 2021 
importance of Blue eyes technological know-how in present day engineering technological expertise - identifying the benefits of using artificial skills technological expertise and the boundaries of artificial intelligence generation. The paper concludes via way of means of the use of spotting benefits of the use of blue eyes generation in current engineering, type of clients that could characteristic the blue eyes generation system, and its effectiveness in current engineering (Gotoa, 2021).

Priyadharsini et al., (2020) proposed a system can discover a human intelligence and reply the reply to the user how it will be that was once the query walking over minds of many scientists. Then later they invented a technological knowhow called BLUE EYES TECHNOLOGY. These blue eyes science can detect the human emotions via a device. It can notice a feeling through eyes, speech recognition, it can additionally apprehend our mood and feelings. It can additionally react to our instructions given by the user. This science created an increase in which human can collaborated to systems. This sensing science in blue eye scan extract the key statistics from the user. The motive for the success of this blue eye's science is that the computer senses the human's feeling (Priyadharsini et al., 2020).

Fiskus et al., (2016) Envision, a stunning worldthe place human beings crew up with Computers! The PC can talk, hear or shriek so everyone may hear! With the assistance of discourse acknowledgment and facial acknowledgment systems, Computers accumulates information from the consumers and begins cooperating with them as indicated via their kingdom of thinking varieties. Computer perceives your passionate degrees by using a basic touch on the mouse and it can speak with us as a shut accomplice. The desktop feels nearness; confirms personality and starts participating with you and even it will dial and name to your domestic at any fundamental circumstances. This all is occurring with this "Blue Eyes" technology (Thakare and Korde, 2020).

Figure 3. 1: Visual Scheme Component Blessed et al. (2018).

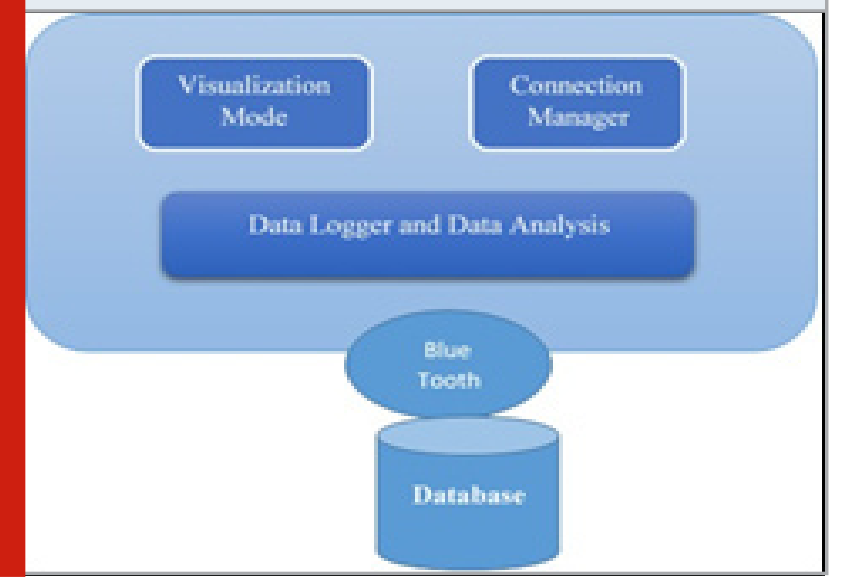

MATERIAL AND METHODS

Blue Eye technology plays vital role in the field of predict the patient's information system with emotional sensors. Blue Eye Technology are classified into three parts such as a) Mobile Measuring Device(MMD) b) Visual Scheme Components(VSC). This paper mainly focused with Visual Scheme Components as shown in the following figure 3.1 below Figure 3. 1: Visual Scheme Component Blessed et al. (2018)

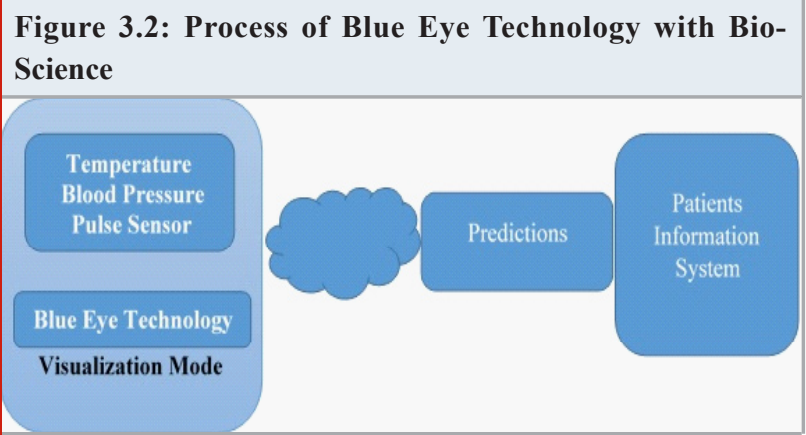

Visual Scheme Component which act as a remote system in the field of Blue eye technology. This Blue eye technology mainly focused with central control processing unit which contain Pulse Code Modulation. This PCM include heartbeat, pulse and Bp used to predict the level using Blue Eye technology in the field of Bio-science to determine the patient's information's. Prediction based information are passed via voice message to database using Blue tooth technology. This Blue Eye Technology used a remote method to find the emotions based sensor of the patients. Three types of sensor such as Temperature Sensor, Pulse Sensor and Blood Sensor are connected with Visualization Mode with Data logger and Data analysis connected with Blue Tooth technology. The Process of this paper as shown in the following Figure 3.2 as below (Blessed et al., 2018; Sainwar et al., 2020; Stieger et al., 2021).

Three Sensor such as Temperature Sensor, Pulse Sensor and Blood Sensor attached with hand using Hand Emotional Sensor. This Hand Emotional Sensor used to determine the temperature, pulse and blood sensor based on the hand movement, this information will be passed to the cloud the used to predict the level of patient's information.

Stepwise Explanation of proposed research work. Algorithm for Predict patient's information using Blue Eye Technology.

\section{Input: Sensor such as Hand Emotion Sensor}

Output: The Level of Patients information using Decision k-NN algorithm

1. Start the process

2. Read the Sensor which attached to the Hand Sensor based on Blue eye emotional sensor system.

3. Emotional Sensor System determine the heart, pulse and blood pressure based on the hand movement by the patient's information systems.

4. Based on the visualization mode, the patient's sensor information will be passed to the database via Blue tooth.

5. Data logger used to predict the patient's information based on the emotional sensor system 
6. Data Analysis focused with K-NN algorithm.

7. Determine the number of $\mathrm{K}$ data set consist of Blood pressure, Temperature and pulse details

8. Find the k-nearest neighbors using basic Euclidian distance formula

9. Determine the membership of k-nearest neighbors with centroid

10. Add the membership with respect to each centroid

11. Assign the class label with maximum membership form the sample data

12. Stop the process.

\section{RESULTS AND DISCUSSION}

A Sample data set as shown in table 5.1 are taken from the hand emotional sensor system which used to predict the information using Bioscience technology. From the above table 5.1, the following graph are generated based on the temperature, heart beat and blood pressure as following in the figure 6.1 .

Table 5.1. Experimental result with Blue Eye Technology

\begin{tabular}{|l|c|c|c|c|}
\hline Patient id & Temperature & $\begin{array}{c}\text { Heart } \\
\text { Beat }\end{array}$ & $\begin{array}{c}\text { Blood } \\
\text { Pressure }\end{array}$ & Status \\
\hline P1 & 21 & 99 & 73 & Good \\
\hline P2 & 22 & 100 & 66 & Good \\
\hline P3 & 10 & 98 & 78 & bad \\
\hline P4 & 33 & 26 & 56 & bad \\
\hline P5 & 55 & 10 & 12 & bad \\
\hline P6 & 53 & 12 & 40 & bad \\
\hline P7 & 21 & 20 & 33 & Good \\
\hline P8 & 18 & 33 & 45 & Good \\
\hline P9 & 15 & 44 & 32 & bad \\
\hline P10 & 44 & 20 & 89 & bad \\
\hline
\end{tabular}

Figure 6.1: Analysis report of Patients Information using Bioscience From the graph, using K-NN algorithm used to predict the patient information status. From the Sainwar et al., (2020) technology and predict the patient's information's (Pillette et al., 2021; Guger et al., 2021).

BLUE EYE TECHNOLOGY - EMOTIONAL SENSOR

nemperature $\quad$ Heart Beat $\quad$ Blood Pressure

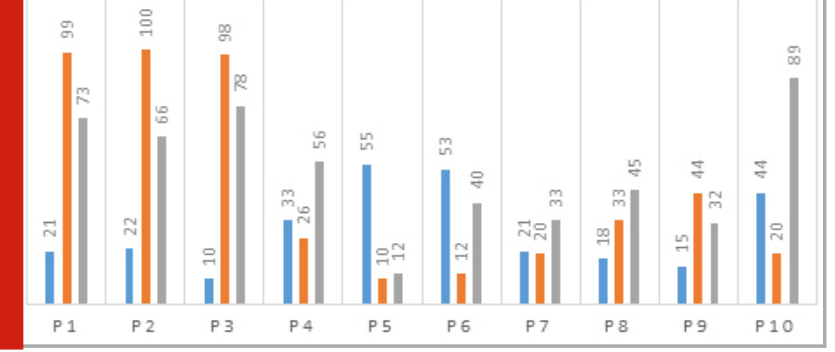

From the graph, using K-NN algorithm used to predict the patient information status. From the Sainwar et al., (2020) technology and predict the patient's information's (Pillette et al., 2021; Guger et al., 2021).

\section{CONCLUSION}

This paper concludes that, the blue eye technology with emotional sensor used to determine the patient's information. This blue eye technology focused with visualization scheme and consist of data logger with data analysis. The Analog data retrieved form the emotional sensor are transmitted to cloud database in the digital form. These digital data will be predicted with K-NN algorithm to determine the patient's information. This paper concluded that an emotional sensor system used to predict the patient's information in the bioscience technology would be effective.

\section{REFERENCES}

Gotoa, R. K. (2021). Development of Training Game Application using Eye-gaze Control Technology to Support Employment of Physically challenged people. Review and Research on Bioscience.

Guger, C., Allison, B.Z. and Gunduz, A., (2021). BrainComputer Interface Research: A State-of-the-Art Summary 10. In Brain-Computer Interface Research (pp. 1-11). Springer, Cham.

Fiskus, W. and Mitsiades, N., (2016). B-Raf inhibition in the clinic: present and future. Annual review of medicine, 67, pp.29-43.

Flesher, S.N., Downey, J.E., Weiss, J.M., Hughes, C.L., Herrera, A.J., Tyler-Kabara, E.C., Boninger, M.L., Collinger, J.L. and Gaunt, R.A., (2021). A brain-computer interface that evokes tactile sensations improves robotic arm control. Science, 372(6544), pp.831-836.

Javaid, M. and Khan, I.H., (2021). Internet of Things (IoT) enabled healthcare helps to take the challenges of COVID19 Pandemic. Journal of Oral Biology and Craniofacial Research, 11(2), pp.209-214.

Oyebola, B. and Toluwani, O., (2018). Blue eyes technology in modern engineering: an artificial intelligence. International Journal of Higher Education, pp.45-65.

Pillette, L., Roc, A., N'kaoua, B. and Lotte, F., (2021). Experimenters' Influence on Mental-Imagery based BrainComputer Interface User Training. International Journal of Human-Computer Studies, 149, p.102603.

Priyadharsini, R. a. (2020). A Comprehensive Study on Blue Eyes Technology Consuming Non-Natural Intellect. International Journal of Engineering Research \& Technology (IJERT).

Reddy, P.C.P., Prashanth, K., Chandana, T.S. and Srikanth, J., (2021). Analyzing Patient Health Information Based on IoT Sensors with AI. Annals of the Romanian Society for Cell Biology, 25(6), pp.15081-15088.

Sainwar, S., Kulshrestha, S. and Sharma, A.K., (2020). Blue Eye Technology. Journal of Advancements in Robotics, 7(1), pp.15-18.

Stieger, J.R., Engel, S.A. and He, B., (2021). Continuous sensorimotor rhythm based brain computer interface learning in a large population. Scientific Data, 8(1), pp.110.

Thakare, A.R. and Korde, N., (2020). Patient Health Monitoring System Using Blue Eye Technology and IoT. Bioscience Biotechnology Research Communications, pp.11-14. 